\title{
A Coalitional Value for Multichoice Games with a Coalition Structure
}

\author{
Fanyong Meng ${ }^{1, *}$ and Qiang Zhang ${ }^{2}$ \\ ${ }^{1}$ School of Management, Qingdao Technological University, Qingdao 266520, China \\ ${ }^{2}$ School of Management and Economics, Beijing Institute of Technology, Beijing 100081, China
}

Received: 6 Jun. 2013, Revised: 8 Oct. 2013, Accepted: 9 Oct. 2013

Published online: 1 Jan. 2014

\begin{abstract}
In this paper a new model of multichoice games with a coalition structure is proposed, which can be seen as an extension of the Owen coalition structure. A coalitional value on the given model is defined, which can be seen as an extension of the Owen value. Three axiomatic systems are studied. The first one is enlightened by Owen's characterization for the Owen value and Faigle and Kern's characterization for the Shapley value on games under precedence constraints. The second one is inspired by Bilbao's characterization for the Shapley value on games on convex geometries. The last one is an extension of Young's characterization for the Shapley value on traditional games. Furthermore, the relationship between the given coalitional value and the core of multichoice games with a coalition structure is discussed.
\end{abstract}

Keywords: multichoice game, coalition structure, Shapley value, core

\section{Introduction}

A multichoice game is a generalization of a traditional TU game in which each player has several activity levels. The reward that a group of players can obtain depends on the efforts of the cooperating players. Hsiao and Raghavan [1] introduced multichoice games in which players have the same number of activity levels, and with each level of activity a weight is associated in order to discriminate between those levels. van den Nouweland et al. [2] considered a more general case with different numbers of activity levels, and extended the notions of core, dominant core and Weber set. They also proposed an alternative extension for the Shapley value based on an extension of the probabilistic formula by orders, but they did not give additional support for this extension. Calvo and Santos [3] studied another value for multichoice games, which is focused on total payoff instead of payoff per level. Klijn et al. [4] have studied a new solution to multichoice games, which is based on the work of Derks and Peters [5]. Calvo and Santos [6] researched a value for multichoice games by restricting the Aumann-Shapley value for continuum games in framework of multichoice games. Peters and Zank [7] proposed the egalitarian solution for multichoice games with each player having the same number of activity levels, which is an extension of the egalitarian solution proposed by Dutta and Ray [8] for traditional games. Recently, Hwang and Liao [9] investigated the weighted associated consistent value, and characterized it by means of the weighted balanced contributions and the associated consistency. More researches can be seen in $[4,6,9,10]$.

As we know, in some cooperative games, such as Economic Community and Military Alliance, the players are joined in coalitions that form a partition or coalitional structure of the set of players. Aumann and Drze [11] first researched in this area and proposed a model of games with a coalition structure, where the players in a union are independent to other players. Different to Aumann and Drze [11], Owen [12] gave another model for games with a coalition structure, where the probability of cooperation among coalitions is considered, and provided the Owen value, which is an extension of the Shapley value. Later, many experts and scholars have studied deeply $[13,14,15$, $16,17,18,19]$.

All above researches are about multichoice games and traditional games with a coalition structure. Both contexts, TU games with a priori system of unions and multichoice games are unified by Albizuri [20] who introduced the concept of a coalition structure for

\footnotetext{
*Corresponding author e-mail: mengfanyongtjie@163.com
} 
multichoice games and defined a multichoice coalition value in this framework. This value is an extension of the Shapley value [21] for traditional games. Based on the coalition structure introduced by Albizuri [20], Jones and Wilson [22] defined another coalitional value in a similar way as the Owen value. Based on the building axiomatic system, the authors showed its existence and uniqueness. From the analysis [22], it shows that the calculation of the introduced coalitional value is complex in the setting of the given model [20] when each player has several participation levels. Further, the coalitional value gives the same weight with respect to the different participation levels. In this paper we introduce another coalition structure for multichoice games, which is an extension of the coalition structure proposed by Owen [12]. According to the works of van den Nouweland et al. [2] and Owen [12], a coalitional value for multichoice games with a coalition structure is proposed, and three axiomatic systems for the given coalitional value are discussed, by which its existence and uniqueness can be proved. When multichoice games are convex, the relationship between the introduced coalitional value and the core is examined, which coincides with the classical case.

This paper is organized as follows. In Section 2, some basic concepts and notions for multichoice games are briefly reviewed. In Section 3, the model of multichoice games with a coalition structure is presented, and a coalitional value is defined. In Section 4, three axiomatic systems for the given coalitional value are discussed. In Section 5, the relationship between the given coalitional value and the core of multichoice games with a coalition structure is examined. The conclusion is made in the last section.

\section{Preliminaries}

Let $N=\{1,2, \ldots, n\}$ be a set of players, and suppose each player $i \in N$ has $m_{i}+1$ activity levels at which he can play. We set $M_{i}=\left\{0,1, \ldots, m_{i}\right\}$ as the action space of player $i \in N$, where the action 0 means no participating, and $M_{i}^{+}=\left\{1, \ldots, m_{i}\right\}$ for any $i \in N$. A function $v: \Pi_{i \in N} M_{i} \rightarrow \mathfrak{R}^{+}$with $v(\emptyset)=0$ gives each coalition $s=\left(s_{1}, s_{2}, \ldots, s_{n}\right) \in M=\prod_{i \in N} M_{i}$ the worth that the players can obtain when each player $i$ plays at level $s_{i} \in M_{i}$. Let $m=\left(m_{1}, m_{2}, \ldots, m_{n}\right)$, and a multichoice game be denoted by a triple $(N, m, v)$, if there is not confusion we will simply denote a multichoice game $(N, m, v)$ by $v$. The set of all multichoice games with the player set $N$ is denoted by $M C^{N}$. For all coalitions $s, t \in M$, let $s \wedge t=\left(s_{i} \wedge t_{i}\right)_{i \in N}$ and $s \vee t=\left(s_{i} \vee t_{i}\right)_{i \in N}$. Furthermore, we denote $s \leq t$ for all $s, t \in M$ if and only if $s_{i} \leq t_{i}$ for each $i \in N$. For any $s=\left(s_{1}, \ldots, s_{n}\right) \in M$, let $S=\left\{i \in N \mid s_{i}>0\right\}$. $e^{S}$ denotes the vector in $N$ satisfying $e_{i}^{S}=1$ for $i \in S$, otherwise, $e_{i}^{S}=0$, where $S \subseteq N$. Especially, $e^{\emptyset}$ denotes the vector in $N$ satisfying $e_{i}^{S}=0$ for all $i \in N$. van den Nouweland et al. [2] gave a value for multichoice games as follows:

$$
\Phi(N, m, v)=\frac{\Pi_{i \in N}\left(m_{i} !\right)}{\left(\sum_{i \in N} m_{i}\right) !} \sum_{\sigma} v^{\sigma},
$$

where $\sigma$ is an admissible order for $v, v^{\sigma}$ means the value of $v$ with respect to the admissible order $\sigma$.

It is not difficult to know that Eq.(1) is equivalent to the following equation.

$$
\begin{array}{r}
\Phi_{i j}(N, m, v)=\sum_{s \in M, s_{i}=j} h_{i j}(s)\left(v(s)-v\left(s-e^{i}\right)\right) \\
\forall i \in N, \quad j \in M_{i}^{+},
\end{array}
$$

where

$h_{i j}(s)=\left(\frac{\left(\sum_{k:\left(s \mid s_{i}-1\right)_{k} \neq 0} s_{k}\right) !}{\Pi_{k:\left(s \mid s_{i}-1\right)_{k} \neq 0}\left(s_{k} !\right)} \frac{\left(\sum_{k \in N}\left(m_{k}-s_{k}\right)\right) !}{\Pi_{k \in N}\left(\left(m_{k}-s_{k}\right) !\right)}\right) / \frac{\left(\sum_{k \in N} m_{k}\right) !}{\Pi_{k \in N}\left(m_{k} !\right)}$, and $s \mid s_{i}-1=\left(s_{1}, \ldots, s_{i-1}, s_{i}-1, s_{i+1}, \ldots, s_{n}\right)$.

$\frac{\left(\sum_{k:\left(s \mid s_{i}-1\right)_{k} \neq 0} s_{k}\right) !}{\Pi_{k:\left(s \mid s_{i}-1\right)_{k} \neq 0}\left(s_{k} !\right)}$ is the number of admissible orders from coalition $e^{\emptyset}$ to coalition $s$, where $s_{i}$ is the last step, and $\frac{\left(\sum_{k \in N}\left(m_{k}-s_{k}\right)\right) !}{\Pi_{k \in N}\left(\left(m_{k}-s_{k}\right) !\right)}$ is the number of admissible orders from coalition $s$ to coalition $m$.

\section{A coalitional value for multichoice games with a coalition structure}

A coalition structure on $(N, m, v)$, given by Albizuri [20], is a family $\mathscr{B}=\left\{B_{1}, \ldots, B_{w}\right\}$ of coalitions on $M$ such that $B_{k}=\left(x_{1}^{k}, x_{2}^{k}, \ldots, x_{n}^{k}\right)$ with $\sum_{k=1}^{w} B_{k}=\left(m_{1}, m_{2}, \ldots, m_{n}\right)$, where $x_{i}^{k}$ denotes the activity level of the player $i$ in coalition $B_{k}$. The author further supposed that the same players do not form two coalitions with different activity levels. However, the author does not give the explanation for this restriction.

Based on the coalition structure introduced by Albizuri [20], Jones and Wilson [22] introduced the following coalitional value, for any $i \in N$, expressed by

$$
\begin{aligned}
\Omega_{i}(N, v, \mathscr{B}) & =\sum_{B_{k} \in A_{i}} \sum_{R \subseteq B_{k} \backslash i} \sum_{S \subseteq W \backslash k} \frac{|S| !(|W|-|S|-1) !}{|W| !} \\
\times \frac{|R| !\left(\left|B_{k}\right|-|R|-1\right) !}{\left|B_{k}\right| !} & \\
& \times\left[v\left(\mathbf{z}+\left(\mathbf{x}_{-i}, x_{i}^{j}\right)\right)-v\left(\mathbf{z}+\left(\mathbf{x}_{-i}, 0\right)\right)\right],
\end{aligned}
$$

where $|S|,|W|,|R|$ and $\left|B_{k}\right|$ respectively denote the cardinalities of $S, W, R$ and $B_{k}, W=\{1,2, \ldots, w\}$, $B_{k}=\left\{i \in N \mid x_{i}^{k} \in M_{i}^{+}\right\}, A_{i}=\left\{B_{k} \mid i \in B_{k}\right\}, \mathbf{z}=\sum_{l \in S} B_{l}$, and $\mathbf{x}_{-i}=\left(\varepsilon_{1} x_{1}^{j}, \ldots, \varepsilon_{i-1} x_{i-1}^{j}, \varepsilon_{i+1} x_{i+1}^{j}, \ldots, \varepsilon_{n} x_{n}^{j}\right) \quad$ with $\varepsilon_{p}=\left\{\begin{array}{cr}1 & p \in R \\ 0 & \text { otherwise }\end{array}\right.$

It is easy to see that the coalitional value degenerates to be the Owen value when each player has only two participation levels 0 and 1. From Eq.(3), we know it is 
focused on total payoff instead of payoff per level. Since the weights given by Eq.(3) only consider the number of active players, it gives the same weight with respect to the different participation levels.

From the coalition structure [20], we know the author considers the player participation levels satisfy addtitivity, e.g., let $M_{i}^{+}=\{1,2\}$, then the player $i$ 's participation level 2 is equal to the sum of his two participation levels 1. This point seems to be unreasonable in some situations. In above example, if these two participation levels of the player $i$ respectively denote a number of money, and the participation levels 1 and 2 respectively denote one million dollars and five million dollars. It is obvious that the participation level 2 is not equal to the sum of two participation levels 1. In the setting of Albizuri's model, the total money of the player $i$ used for cooperation is two million dollars rather than five million dollars.

Different to the coalition structure given by Albizuri [20], we introduce another coalition structure on multichoice games. Owen [12] gave a coalition structure for traditional games as follows:

Definition 3.1. [12] $\Gamma=\left\{B_{1}, B_{2}, \ldots, B_{h}\right\}$ is said to be a coalition structure on player set $N$, if it satisfies $\cup_{1 \leq k \leq h} B_{k}=N$ and $B_{k} \cap B_{l}=\emptyset$ for all $k \neq l$, where $k, l \in P=\{1,2, \ldots, h\}$.

Here, we apply the coalition structure introduced by Owen [12] in the framework of multichoice games. For any given $B_{k} \in \Gamma$, let $b_{k} \in M$ denote the "maximum coalition" on $B_{k}$, namely, $\left(b_{k}\right)_{i}=m_{i}$ for all $i \in B_{k}$, and $\left(b_{k}\right)_{i}=0$, otherwise. Then $\left\{b_{1}, b_{2}, \ldots, b_{h}\right\}$ is a partition of $m$. Let $(N, m, \Gamma)$ denote a coalition structure on $(N, m)$.

Definition 3.2. $s \in M$ is said to be a feasible coalition for $(N, m, \Gamma)$, if there exist $B_{k} \in \Gamma$ and $\cup_{l \in R \subseteq P \backslash k} B_{l}$ such that $s=t_{1} \vee t_{2}$, where $t_{1} \leq b_{k}$ and $t_{2}=\vee_{l \in R \subseteq P \backslash k} b_{l}$.

Let $\mathrm{Fe}(N, m, \Gamma)$ be the set of all feasible coalitions in $(N, m, \Gamma)$. From Definition 3.2, it is easy to see when the coalition structure $(N, m, \Gamma)$ is restricted in the setting of the model given in [12], then $\mathrm{Fe}(N, m, \Gamma)$ degenerates to be the set of feasible coalitions for $\Gamma$ on $N$.

Example 3.1. Let the player set $N=\{1,2,3,4\}$, $m=(2,2,2,1)$ and $\Gamma=\left\{B_{1}, B_{2}\right\}$, where $B_{1}=\{1,2\}$ and $B_{2}=\{3,4\}$. For $B_{1}$ and $s=(1,2,0,0) \leq b_{1}$, when the players 1 and 2 cooperate with the players 3 and 4 , then $s^{\prime}=(1,2,2,1)$ is the only feasible coalition with respect to $s$.

Example 3.2. Let the player set $N=\{1,2,3\}$, $m=(2,2,1)$ and $\Gamma=\left\{B_{1}, B_{2}\right\}$, where $B_{1}=\{1\}$ and $B_{2}=\{2,3\}$, then $F e(N, m, \Gamma)=\{(1,0,0),(0,1,0),(0$, $0,1),(2,0,0)(0,2,0),(0,1,1),(0,2,1),(2,1,0),(2,2,0)$, $(2,0,1),(1,2,1),(2,1,1),(2,2,1)\}$.

Definition 3.3. Let $v \in M C^{N}, t \in M$ is said to be a carrier for $v$ in $M$ if $v(s)=v(s \wedge t)$ for all $s \in M$.

Definition 3.4. Let $v \in M C^{N}$ and $\Gamma=\left\{B_{1}, B_{2}, \ldots, B_{h}\right\} \cdot v^{B}$ is said to be a quotient game on $\Gamma$, if it satisfies $v^{B}(R)=$ $v\left(\vee_{l \in R} b_{l}\right)$ for all $R \subseteq P=\{1,2, \ldots, h\}$.
Definition 3.3 is an extension of the carrier proposed by Shapley [21], and Definition 3.4 is an extension of the quotient game introduced by Owen [12].

Let $(N, m, v, \Gamma)$ be a multichoice game with the coalition structure $\Gamma$, and $M C^{C N}$ be the set of all multichoice games with a coalition structure. Following the works of van den Nouweland et al. [2] and Owen [12], we introduce a coalitional value on $M C^{C N}$ as follows:

$$
\begin{array}{r}
\psi_{i j}(N, m, v, \Gamma)=\sum_{R \subseteq H \backslash k \leq b_{k}, s_{i}=j} \frac{r !(h-r-1) !}{h !} h_{i j}^{B_{k}}(s) \\
\left(v(s \vee t)-v\left(s \vee t-e^{i}\right)\right) \forall i \in N, \quad j \in M_{i}^{+},
\end{array}
$$

where $p$ and $r$ respectively denote the cardinalities of $P$ and $R$, and $t=\vee_{w \in R} b_{w} . h_{i j}^{B_{k}}(s)$ is the coefficient for the coalition $s$ restricted on $B_{k}$. Namely, $h_{i j}^{B_{k}}(s)=$ $\left(\frac{\left(\sum_{g:\left(s \mid s_{i}-1\right)_{g} \neq 0} s_{g}\right) !}{\Pi_{g:\left(s \mid s_{i}-1\right)_{g} \neq 0}\left(s_{g} !\right)} \frac{\left(\sum_{g \in B_{k}}\left(m_{g}-s_{g}\right)\right) !}{\Pi_{g \in B_{k}}\left(\left(m_{g}-s_{g}\right) !\right)}\right) / \frac{\left(\sum_{g \in B_{k}} m_{g}\right) !}{\Pi_{g \in B_{k}}\left(m_{g} !\right)} \quad$ with $s \mid s_{i}-1=\left(s_{k_{1}}, \ldots, s_{i}-1, \ldots, s_{k_{t}}\right)$ and $\left\{k_{1}, \ldots, i, \ldots, k_{t}\right\} \subseteq B_{k}$.

Obviously, Eq.(4) degenerates to be the Owen value [12] when $v$ is restricted in the setting of games with a coalition structure, namely, every player has only two activity levels 0 and 1. Furthermore, Eq.(4) degenerates to be the Shapley value for multichoice games [2] when $h$ is equal to 1 , namely, there is only one coalition in $\Gamma$, that is $N$. In other words, Eq.(4) can be seen as an extension both of them.

As we know, when a coalition structure for traditional games has $n$ coalitions, the Owen value degenerates to be the Shapley value for traditional games. But this point does not hold any more when it turns to multichoice games. Namely, when a coalition structure $\Gamma$ for a multichoice game has $n$ coalitions, it can not guarantee the given coalitional value is equal to the Shapley value introduced by van den Nouweland et al. [2].

Example 3.3. Let $N=\{1,2\}, \quad m=(2,2)$ and $\Gamma=\left\{B_{1}, B_{2}\right\}$, where $B_{1}=\{1\}$ and $B_{2}=\{2\}$. If the values for coalitions are given as follows: $v(1,0)=v(0,1)$ $=1, v(2,0)=v(0,2)=3, v(1,1)=5, v(1,2)=8$, $v(2,1)=10, v(2,2)=15$.

From Eq.(4), it has

$$
\begin{aligned}
& \psi_{11}(N, m, v, \Gamma)=3, \\
& \psi_{12}(N, m, v, \Gamma)=4.5, \\
& \psi_{21}(N, m, v, \Gamma)=4, \\
& \psi_{22}(N, m, v, \Gamma)=3.5 .
\end{aligned}
$$

From Eq.(2), it gets

$$
\begin{aligned}
& \phi_{11}(N, m, v)=2.67 \\
& \phi_{12}(N, m, v)=5.5, \\
& \phi_{21}(N, m, v)=3 \\
& \phi_{22}(N, m, v)=3.83 .
\end{aligned}
$$

From above, we know $\psi \neq \phi$. 


\section{Characterizations of the given coalitional value}

In this section, we mainly discuss the axiomatic systems of the given coalitional value. Let $\varphi$ be a coalitional value on $M C^{C N}$.

The analogue of unanimity games for multichoice games are minimal effort games $\left(N, m, u_{s}\right) \in M C^{N}$, for any $s \in M \backslash\left\{e^{\emptyset}\right\}$, defined by

$$
u_{s}(t)=\left\{\begin{array}{lc}
1 & s \leq t \\
0 & \text { otherwise }
\end{array}\right.
$$

for all $t \in M \backslash\left\{e^{\emptyset}\right\}$.

The identity games for multichoice games are games $\left(N, m, \delta_{s}\right) \in M C^{N}$, for any $s \in M \backslash\left\{e^{\emptyset}\right\}$, defined by

$$
u_{s}(t)=\left\{\begin{array}{lc}
1 & s=t \\
0 & \text { otherwise }
\end{array}\right.
$$

for all $t \in M \backslash\left\{e^{\emptyset}\right\}$.

According to the literature [12,23], we give the following properties for the coalitional value $\varphi$.

additivity $(\boldsymbol{A D D})$. Let $v, w \in M C^{C N}$, then

$$
\varphi(N, m, v+w, \Gamma)=\varphi(N, m, v, \Gamma)+\varphi(N, m, w, \Gamma) .
$$

efficiency $(\boldsymbol{E F F})$. Let $v \in M C^{C N}$, then

$$
\sum_{i \in N} \sum_{j=1}^{m_{i}} \varphi_{i j}(N, m, v, \Gamma)=v(m) .
$$

null property (NP). Let $v \in M C^{C N}$ and $i \in N$, if $j \in M_{i} \backslash\left\{e^{\emptyset}\right\}$ is a "null level", then $\varphi_{i j}(N, m, v, \Gamma)=0$. Here, $j \in M_{i} \backslash\left\{e^{\emptyset}\right\}$ is said to be a "null level" if $v\left(s \vee(j-1) e^{i}\right)=v\left(s \vee j e^{i}\right)$ for all $s \in \Pi_{k \in N \backslash\{i\}} M_{k}$. hierarchical strength within coalition (HST). Let $v \in M C^{C N}$. For $B_{k} \in \Gamma$ and all $i, j \in B_{k}$, it has

$$
\begin{aligned}
& \sum_{\substack{s \wedge b_{k} \leq x \leq b_{k}, x_{j}=s_{j}}} h_{j s_{j}}^{B_{k}}(x) \varphi_{i s_{i}}\left(N, m, u_{s}, \Gamma\right) \\
= & \sum_{\substack{s \wedge b_{k} \leq x \leq b_{k} \\
x_{i}=s_{i}}} h_{i s_{i}}^{B_{k}}(x) \varphi_{j s_{j}}\left(N, m, u_{s}, \Gamma\right)
\end{aligned}
$$

for any $s \in F e(N, m, \Gamma)$ with $s_{i} \in M_{i}^{+}$and $s_{j} \in M_{j}^{+}$.

symmetry in quotient game $(\boldsymbol{S Q})$. Let $v \in M C^{C N}$. For all $B_{k}, B_{s} \in \Gamma$, if $v^{B}(R \cup k)=v^{B}(R \cup s)$ for any $R \subseteq P \backslash\{k, s\}$, then

$$
\sum_{i \in B_{k}} \sum_{l=1}^{m_{i}} \varphi_{i l}(N, m, v, \Gamma)=\sum_{j \in B_{s}} \sum_{h=1}^{m_{j}} \varphi_{j h}(N, m, v, \Gamma) .
$$

Remark 4.1. The properties $\boldsymbol{A D D}, \boldsymbol{E F F}, \boldsymbol{N P}$ and $S Q$ degenerate to be the properties introduced by Owen [12] when we restrict the domain of $C M^{C N}$ in the setting of traditional games with a coalition structure. Furthermore, the property $\boldsymbol{H S T}$ degenerates to be the property proposed by Faigle and Kern [23] when the domain of $C M^{C N}$ is limited to traditional games.

Theorem 4.1. A coalitional value satisfies $\boldsymbol{A D D}, \boldsymbol{E F F}$, $\boldsymbol{N P}, \boldsymbol{H S T}$ and $\boldsymbol{S Q}$ if, and only if, it is equal to Eq.(4), i.e., $\varphi_{i j}(N, m, v, \Gamma)=\psi_{i j}(N, m, v, \Gamma)$ for all $i \in N$ and all $j \in M_{i}^{+}$, where $v \in M C^{C N}$.

Proof. First we show that $\psi$ satisfies these properties. $\psi$ satisfies $A D D$ and $N P$ as is easily seen with Eq.(4).

In the following, we shall $\psi$ show satisfies the rest characterizations.

From Eq.(4), it has

$$
\begin{aligned}
& \sum_{i=1}^{N} \sum_{j=1}^{m_{i}} \psi_{i j}(N, m, v, \Gamma) \\
& =\sum_{i=1}^{N} \sum_{j=1}^{m_{i}} \sum_{R \subseteq P \backslash k s \leq b_{k}, s_{i}=j} \frac{r !(p-r-1) !}{p !} \\
& \quad \times h_{i j}^{B_{k}}(s)\left(v(s \vee t)-v\left(s \vee t-e^{i}\right)\right) .
\end{aligned}
$$

For any $s \leq b_{k}$, let $v^{t}(s)=v(s)-v(t)$. It gets

$$
\begin{aligned}
& \sum_{i=1}^{N} \sum_{j=1}^{m_{i}} \psi_{i j}(N, m, v, \Gamma) \\
&=\sum_{i=1}^{N} \sum_{j=1}^{m_{i}} \sum_{R \subseteq P \backslash k s \leq b_{k}, s_{i}=j} \frac{r !(p-r-1) !}{p !} \\
& \quad \times h_{i j}^{B_{k}}(s)\left(v(s \vee t)-v\left(s \vee t-e^{i}\right)\right) \\
&=\sum_{k \in P R \subseteq P \backslash k} \frac{r !(p-r-1) !}{p !} v^{t}\left(b_{k}\right) \\
&=\sum_{k \in P R \subseteq P \backslash k} \frac{r !(p-r-1) !}{p !}\left(v\left(b_{k} \vee t\right)-v(t)\right) \\
&=\sum_{k \in P R \subseteq P \backslash k} \frac{r !(p-r-1) !}{p !}\left(v^{B}(R \cup k)-v^{B}(R)\right) \\
&=v^{B}(P) \\
&= v\left(\vee_{k \in P} b_{k}\right) \\
&= v(m) .
\end{aligned}
$$

From above, it shows $\boldsymbol{E F F}$ holds.

From Eq.(4) and the conditions in $\boldsymbol{H S T}$, it gets

$$
\begin{aligned}
& \psi_{i s_{i}}\left(N, m, u_{s}, \Gamma\right) \\
& =\sum_{R \subseteq P \backslash k x \leq b_{k}, x_{i}=s_{i}} \frac{r !(p-r-1) !}{p !} h_{i s_{i}}^{B_{k}}(x) \\
& \quad \times\left(u_{s}(x \vee t)-u_{s}\left(x \vee t-e^{i}\right)\right) \\
& =\sum_{P^{\prime} \backslash k \subseteq R \subseteq P \backslash k s \wedge b_{k} \leq x \leq b_{k}, x_{i}=s_{i}} \frac{r !(p-r-1) !}{p !} h_{i s_{i}}^{B_{k}}(x)
\end{aligned}
$$




$$
\begin{aligned}
& =\sum_{P^{\prime} \backslash k \subseteq R \subseteq P \backslash k} \frac{r !(p-r-1) !}{p !} \sum_{s \wedge b_{k} \leq x \leq b_{k}, x_{i}=s_{i}} h_{i s_{i}}^{B_{k}}(x) \\
& =\frac{1}{p^{\prime}} \sum_{s \wedge b_{k} \leq x \leq b_{k}, x_{i}=s_{i}} h_{i s_{i}}^{B_{k}}(x),
\end{aligned}
$$

where $\frac{1}{p^{\prime}}=\sum_{P^{\prime} \backslash k \subseteq R \subseteq P \backslash k} \frac{r !(p-r-1) !}{p !}$ and $p^{\prime}$ is the cardinality of $P^{\prime}=\left\{l \in P \mid b_{l} \wedge s \neq e^{\emptyset}\right\}$.

For the same reason, it has

$$
\psi_{j s_{j}}\left(N, m, u_{s}, \Gamma\right)=\frac{1}{p^{\prime}} \sum_{s \wedge b_{k} \leq x \leq b_{k}, x_{j}=s_{j}} h_{j s_{j}}^{B_{k}}(x) .
$$

Now one can easily verify that $\psi$ indeed satisfies the property of $\boldsymbol{H S T}$.

From the proof of $\boldsymbol{E F F}$, it has

$$
\begin{aligned}
& \sum_{i \in B_{k}} \sum_{l=1}^{m_{i}} \psi_{i l}(N, m, v, \Gamma) \\
& =\sum_{R \subseteq P \backslash k} \frac{r !(p-r-1) !}{p !}\left(v^{B}(R \cup k)-v^{B}(R)\right)
\end{aligned}
$$

and

$$
\begin{aligned}
& \sum_{j \in B_{s}} \sum_{h=1}^{m_{i}} \psi_{j h}(N, m, v, \Gamma) \\
& =\sum_{R \subseteq P \backslash s} \frac{r !(p-r-1) !}{p !}\left(v^{B}(R \cup s)-v^{B}(R)\right) .
\end{aligned}
$$

Since, $v^{B}(R \cup k)=v^{B}(R \cup s)$ for all $R \subseteq P \backslash\{k, s\}$, it gets $S Q$.

Uniqueness. Let $\varphi$ be a coalitional value on $M C^{C N}$, and satisfy the above mentioned properties. In the following, we shall show $\varphi_{i j}(N, m, v, \Gamma)=\left\{\psi_{i j}\right\}(N, m, v, \Gamma)$ for all $i \in$ $N$ and all $j \in M_{i}^{+}$.

From Lemma 3.1 in Derks and Peters [5] and $\boldsymbol{A D D}$, it is sufficient to show that $\varphi$ and $\psi$ coincides on the class of the analogue of unanimity games. For any $s \in \mathrm{Fe}(N, m, \Gamma)$, let $P^{\prime}=\left\{l \in P \mid b_{l} \wedge s \neq e^{\emptyset}\right\}$ and $B_{l}^{\prime}=B_{l} \cap S$. Define quotient game $\left(P, u_{s}^{P}\right)$ as follows:

$$
u_{s}^{B}(R)=\left\{\begin{array}{rr}
1 & P^{\prime} \subseteq R \\
0 & \text { otherwise }
\end{array}\right.
$$

for all $R \subseteq P$.

Since, $\varphi$ satisfies $\boldsymbol{N P}$, we get $\varphi_{i j}\left(N, m, u_{s}, \Gamma\right)=0$ for all $i \in N \backslash S$ with $j \in M_{i}^{+}$, and all $i \in S$ with $j \in M_{i}^{+}$and $j \neq s_{i}$. From $\boldsymbol{E F F}, \boldsymbol{N P}$ and $\boldsymbol{S Q}$, it gets

$$
\sum_{i \in \dot{B}_{k}} \varphi_{i s_{i}}\left(N, m, u_{s}, \Gamma\right)=\left\{\begin{array}{cc}
\frac{1}{p^{\prime}} & k \in P^{\prime} \\
0 & k \notin P^{\prime}
\end{array}\right.
$$

for all $i \in S$ with $j=s_{i} \in M_{i}^{+}$.

From $\boldsymbol{E F F}$ and $\boldsymbol{H S T}$, it has

$\varphi_{i j}\left(N, m, u_{s}, \Gamma\right)=\left\{\begin{array}{cl}\frac{1}{p^{\prime}} \sum_{s \wedge b_{k} \leq x \leq b_{k}, x_{i}=s_{i}} h_{i s_{i}}^{B_{k}}(x) j=s_{i} \in M_{i}^{+} \\ 0 & \text { otherwise }\end{array}\right.$.
On the other hand, from Eq.(4) it gets

$$
\psi_{i j}\left(N, m, u_{s}, \Gamma\right)=\left\{\begin{array}{cc}
\frac{1}{p^{\prime}} \sum_{s \wedge b_{k} \leq x \leq b_{k}, x_{i}=s_{i}} h_{i s_{i}}^{B_{k}}(x) j=s_{i} \in M_{i}^{+} \\
0 \quad \text { otherwise }
\end{array} .\right.
$$

Hence, $\varphi\left(N, m, u_{s}, \Gamma\right)=\psi\left(N, m, u_{s}, \Gamma\right)$. From the randomicity of $s \in \mathrm{Fe}(N, m, \Gamma)$, the desired result is obtained.

linearity $(\boldsymbol{L})$. Let $v, w \in M C^{C N}$ and $\alpha, \beta \in \Re$, then

$$
\varphi(N, m, \alpha v+\beta w, \Gamma)=\alpha \varphi(N, m, v, \Gamma)+\beta \varphi(N, m, w, \Gamma) .
$$

carrier $(\boldsymbol{C})$. If $t \in M$ is a carrier for the multichoice game $v \in M C^{C N}$, then

$$
\sum_{i \in T} \sum_{j=1}^{t_{i}} \varphi_{i j}(N, m, v, \Gamma)=v(t)
$$

where $T=\left\{k \mid t_{k} \neq 0, k \in N\right\}$.

When we restrict the domain of $M C^{C N}$ in the setting of traditional games, the property $\boldsymbol{C}$ degenerates to be the property proposed by Shapley [21].

Remark 4.2. From Theorem 4.1, we know Eq.(4) is the unique coalitional value on $M C^{C N}$ that satisfies $\boldsymbol{A D D}, \boldsymbol{C}$, $\boldsymbol{H S T}$ and $\boldsymbol{S Q}$, as well as the unique coalitional value on $M C^{C N}$ that satisfies $\boldsymbol{L}, \boldsymbol{C}, \boldsymbol{H S T}$ and $\boldsymbol{S Q}$.

Bilbao [24] gave a characterization of the Shapley value for games on convex geometries by using chain axiom. Here, we define it in the setting of multichoice games with a coalition structure and give the following property.

chain axiom $(\boldsymbol{C A})$. Let $v \in M C^{C N}$. For all $B_{k} \in \Gamma$ and all $i, j \in B_{k}$ with $s_{i} \in M_{i}^{+}$and $s_{j} \in M_{j}^{+}$, then

$$
\begin{aligned}
& c^{B_{k}}\left[0, s \mid s_{i}-1\right] \varphi_{j s_{j}}\left(N, m, \delta_{s}, \Gamma\right) \\
& =c^{B_{k}}\left[0, s \mid s_{j}-1\right] \varphi_{i s_{i}}\left(N, m, \delta_{s}, \Gamma\right)
\end{aligned}
$$

and

$$
\begin{aligned}
& \left(\left(p^{\prime}+l-1\right) !\left(p-p^{\prime}-l\right) !\right) \varphi_{i s_{i}}\left(N, m, \delta_{s}, \Gamma\right) \\
& =\left(\left(p^{\prime}-1\right) !\left(p-p^{\prime}\right) !\right) \varphi_{i s_{i}}\left(N, m, \delta_{s^{\prime}}, \Gamma\right)
\end{aligned}
$$

where $s, s^{\prime} \in F e(N, m, \Gamma)$ such that $s \vee_{r=0}^{l} b_{r}=s^{\prime}$ and $s \wedge\left(\bigvee_{r=0}^{l} b_{r}\right)=e^{\emptyset} . s \mid s_{i}-1$ and $s \mid s_{j}-1$ as shown in Eq.(4). $c^{B_{k}}\left[0, s \mid s_{i}-1\right]=\frac{\left(\sum_{g:\left(s \mid s_{i}-1\right)_{g} \neq 0} s_{g}\right) !}{\Pi_{g:\left(s \mid s_{i}-1\right)_{g} \neq 0}\left(s_{g} !\right)} \quad$ and $c^{B_{k}}\left[0, s \mid s_{j}-1\right]$ $=\frac{\left(\sum_{g:\left(s \mid s_{j}-1\right)_{g} \neq 0} s_{g}\right) !}{\Pi_{g:\left(s \mid s_{j}-1\right)_{g} \neq 0}\left(s_{g} !\right)} \cdot p^{\prime}$ is the cardinality of $P^{\prime}=\{l \in P \mid$ $\left.b_{l} \wedge s \neq e^{\emptyset}\right\}$.

It is obviously that the chain axiom on $M C^{C N}$ degenerates to be the property introduced by Bilbao [24] when we restrict the domain of $M C^{C N}$ in the setting of traditional games on convex geometries.

Theorem 4.2. There is a unique solution $\varphi$ defined on $M C^{C N}$ that satisfies $\boldsymbol{L}, \boldsymbol{E F F}, \boldsymbol{N P}, \boldsymbol{C A}$ and $S Q$, i.e., $\varphi_{i j}(N, m, v, \Gamma)=\psi_{i j}(N, m, v, \Gamma)$ for all $i \in N$ and all $j \in M_{i}^{+}$. 
Proof. From Theorem 4.1 and Eq.(4), we know $\psi$ satisfies $\boldsymbol{L}, \boldsymbol{E F F}, \boldsymbol{N P}$ and $\boldsymbol{S Q}$. In the following, we will show $\psi$ satisfies $\boldsymbol{C A}$. From the definition of $\delta_{s}$, it gets

$$
\begin{aligned}
\psi_{i s_{i}}\left(N, m, \delta_{s}, \Gamma\right) & =\frac{\left(p^{\prime}-1\right) !\left(p-p^{\prime}\right) !}{p !} h_{i s_{i}}^{B_{k}}\left(s \wedge b_{k}\right) \\
& =\gamma \frac{\left(p^{\prime}-1\right) !\left(p-p^{\prime}\right) !}{p !} c^{B_{k}}\left[0, s \mid s_{i}-1\right],
\end{aligned}
$$

where $\gamma=\frac{\left(\sum_{g \in B_{k}}\left(m_{g}-s_{g}\right)\right) !}{\Pi_{g \in B_{k}}\left(\left(m_{g}-s_{g}\right) !\right)} / \frac{\left(\sum_{g \in B_{k}} m_{g}\right) !}{\Pi_{g \in B_{k}}\left(m_{g} !\right)}$

Similarly, it has

$$
\psi_{j s_{j}}\left(N, m, \delta_{s}, \Gamma\right)=\gamma \frac{\left(p^{\prime}-1\right) !\left(p-p^{\prime}\right) !}{p !} c^{B_{k}}\left[0, s \mid s_{j}-1\right] .
$$

Thus,

$$
\begin{aligned}
& c^{B_{k}}\left[0, s \mid s_{i}-1\right] \varphi_{j s_{j}}\left(N, m, \delta_{s}, \Gamma\right) \\
& =c^{B_{k}}\left[0, s \mid s_{j}-1\right] \varphi_{i s_{i}}\left(N, m, \delta_{s}, \Gamma\right) .
\end{aligned}
$$

From above, it shows

$$
\psi_{i s_{i}}\left(N, m, \delta_{s}, \Gamma\right)=\frac{\left(p^{\prime}-1\right) !\left(p-p^{\prime}\right) !}{p !} h_{i s_{i}}^{B_{k}}\left(s \wedge b_{k}\right)
$$

and

$$
\psi_{i s_{i}}\left(N, m, \delta_{s^{\prime}}, \Gamma\right)=\frac{\left(p^{\prime}+l-1\right) !\left(p-p^{\prime}-l\right) !}{p !} h_{i s_{i}}^{B_{k}}\left(s^{\prime} \wedge b_{k}\right) .
$$

Since $h_{i s_{i}}^{B_{k}}\left(s^{\prime} \wedge b_{k}\right)=h_{i s_{i}}^{B_{k}}\left(s \wedge b_{k}\right)$, it gets

$$
\begin{aligned}
& \left(\left(p^{\prime}+l-1\right) !\left(p-p^{\prime}-l\right) !\right) \varphi_{i s_{i}}\left(N, m, \delta_{s}, \Gamma\right) \\
& =\left(\left(p^{\prime}-1\right) !\left(p-p^{\prime}\right) !\right) \varphi_{i s_{i}}\left(N, m, \delta_{s^{\prime}}, \Gamma\right) .
\end{aligned}
$$

Uniqueness. Let $\varphi$ be a coalitional value on $M C^{C N}$, and satisfy the above mentioned properties. For any $v \in M C^{C N}$, there exists an unique set of coefficients $\{v(s): s \in F e(N, m, \Gamma)\}$ such that $v=\sum_{s \in F e(N, m, \Gamma)} v(s) \delta_{s}$. From $L$, we only need to prove $\varphi$ and $\psi$ coincides on $\left(N, m, \delta_{t}, \Gamma\right)$ for any $t \in F e(N, m, \Gamma)$. Similar to Theorem 4.1, for any $s \in F e(N, m, \Gamma)$, let $P^{\prime}=\left\{l \in P \mid b_{l} \wedge s \neq e^{\emptyset}\right\}$ and $B_{l}^{\prime}=B_{l} \cap S$. Define quotient game $\left(P, u_{s}^{B}\right)$ as follows:

$$
u_{s}^{B}(R)=\left\{\begin{array}{cc}
1 & P^{\prime} \subseteq R \\
0 & \text { otherwise }
\end{array}\right.
$$

for all $R \subseteq P$.

From Theorem 4.1, it gets

$$
\sum_{i \in B_{k}} \varphi_{i s_{i}}\left(N, m, u_{s}, \Gamma\right)=\left\{\begin{array}{cc}
\frac{1}{p^{\prime}} & k \in P^{\prime} \\
0 & k \notin P^{\prime}
\end{array}\right.
$$

Since $u_{s}=\sum_{s \leq t} \delta_{t}$ for all $s \in F e(N, m, \Gamma)$, it has

$$
\begin{aligned}
\varphi\left(N, m, u_{s}, \Gamma\right) & =\varphi\left(N, m, \sum_{s \leq t} \delta_{t}, \Gamma\right) \\
& =\sum_{s \leq t} \varphi\left(N, m, \delta_{t}, \Gamma\right)
\end{aligned}
$$

and

$$
\varphi_{i s_{i}}\left(N, m, u_{s}, \Gamma\right)=\sum_{s \leq t, t_{i}=s_{i}} \varphi_{i s_{i}}\left(N, m, \delta_{t}, \Gamma\right)
$$

From $\boldsymbol{C A}$, it gets

$$
\begin{aligned}
& \varphi_{i s_{i}}\left(N, m, u_{s}, \Gamma\right) \\
= & \sum_{\substack{s \wedge b_{k} \leq t \wedge b_{k}, t_{i}=s_{i}, t-b_{k}=s-b_{k}}}\left(\sum_{0 \leq l \leq p-p^{\prime}} \frac{\left(p^{\prime}+l-1\right) !\left(p-p^{\prime}-l\right) !}{\left(p^{\prime}-1\right) !\left(p-p^{\prime}\right) !}\right) \\
& \times \varphi_{i s_{i}}\left(N, m, \delta_{t}, \Gamma\right) \\
= & \left.\sum_{0 \leq l \leq p-p^{\prime}} \frac{\left(p^{\prime}+l-1\right) !\left(p-p^{\prime}-l\right) !}{\left(p^{\prime}-1\right) !\left(p-p^{\prime}\right) !}\right) \sum_{\substack{s \wedge b_{k} \leq t \wedge b_{k}, t_{i}=s_{i}, t-b_{k}=s-b_{k}}} \sum_{=} \frac{p \varphi_{i s_{i}}\left(N, m, \delta_{t}, \Gamma\right)}{p^{\prime}} \frac{p !}{\left(p^{\prime}-1\right) !\left(p-p^{\prime}\right) !} \sum_{\substack{s \wedge b_{k} \leq t \wedge b_{k}, t_{i}=s_{i}, t-b_{k}=s-b_{k}}} \varphi_{i s_{i}}\left(N, m, \delta_{t}, \Gamma\right) .
\end{aligned}
$$

From above, we know $P^{\prime}=\left\{l \in P \mid b_{l} \wedge s \neq e^{\emptyset}\right\}=\{l \in$ $\left.P \mid b_{l} \wedge t \neq e^{\emptyset}\right\}$.

From $\boldsymbol{C A}$, it has

$$
h_{i t_{i}}^{B_{k}}(t) \varphi_{j t_{j}}\left(N, m, \delta_{t}, \Gamma\right)=h_{j t_{j}}^{B_{k}}(t) \varphi_{i t_{i}}\left(N, m, \delta_{t}, \Gamma\right),
$$

which is equal to

$h_{i t_{i}}^{B_{k}}\left(t \wedge b_{k}\right) \varphi_{j t_{j}}\left(N, m, \delta_{t}, \Gamma\right)=h_{j t_{j}}^{B_{k}}\left(t \wedge b_{k}\right) \varphi_{i t_{i}}\left(N, m, \delta_{t}, \Gamma\right)$.

Namely,

$$
\varphi_{i t_{i}}\left(N, m, \delta_{t}, \Gamma\right)=\frac{h_{i t_{i}}^{B_{k}}\left(t \wedge b_{k}\right)}{h_{j t_{j}}^{B_{k}}\left(t \wedge b_{k}\right)} \varphi_{j t_{j}}\left(N, m, \delta_{t}, \Gamma\right) .
$$

By Eq.(6), it has

$$
\begin{aligned}
\frac{1}{p^{\prime}}= & \frac{1}{p^{\prime}} \frac{p !}{\left(p^{\prime}-1\right) !\left(p-p^{\prime}\right) !} \\
& \times \sum_{i \in \dot{B}_{k}} \sum_{\substack{s \wedge b_{k} \leq t \wedge b_{k}, t_{i}=s_{i}, t-b_{k}=s-b_{k}}} \varphi_{i s_{i}}\left(N, m, \delta_{t}, \Gamma\right)
\end{aligned}
$$

and

$$
\begin{aligned}
& \frac{\left(p^{\prime}-1\right) !\left(p-p^{\prime}\right) !}{p !} \\
& =\sum_{i \in \dot{B}_{k}} \sum_{\substack{s \wedge b_{k} \leq t \wedge b_{k}, t_{i}=s_{i}, t-b_{k}=s-b_{k}}} \varphi_{i s_{i}}\left(N, m, \delta_{t}, \Gamma\right) .
\end{aligned}
$$

When $\sum_{i \in S \cap B_{k}} s_{i}=\sum_{i \in B_{k}}\left(b_{k}\right)_{i}$, by Eqs.(6) and (7), it gets

$$
\frac{\left(p^{\prime}-1\right) !\left(p-p^{\prime}\right) !}{p !}
$$




$$
\begin{aligned}
& =\sum_{\substack{i \in B_{k} \\
\text { s^b } \\
b_{k}, t_{j}=s_{j}=m_{j}}} \varphi_{i s_{i}}\left(N, m, \delta_{t}, \Gamma\right) \\
& =\varphi_{j m_{j}}\left(N, m, \delta_{t}, \Gamma\right)+\sum_{i \in B_{k} \backslash j} \frac{h_{i m_{i}}^{B_{k}}\left(b_{k}\right)}{h_{j m_{j}}^{B_{k}}\left(b_{k}\right)} \varphi_{j m_{j}}\left(N, m, \delta_{t}, \Gamma\right) \\
& =\frac{\sum_{i \in B_{k}} h_{i m_{i}}^{B_{k}}\left(b_{k}\right)}{h_{j m_{j}}^{B_{k}}\left(b_{k}\right)} \varphi_{j m_{j}}\left(N, m, \delta_{t}, \Gamma\right) .
\end{aligned}
$$

Since $\sum_{i \in B_{k}} h_{i m_{i}}^{B_{k}}\left(b_{k}\right)=1$, it has

$$
\varphi_{j m_{j}}\left(N, m, \delta_{t}, \Gamma\right)=\frac{\left(p^{\prime}-1\right) !\left(p-p^{\prime}\right) !}{p !} h_{j m_{j}}^{B_{k}}\left(b_{k}\right)
$$

for all $j \in B_{k}$.

When $\sum_{i \in S \cap B_{k}} s_{i}=\sum_{i \in B_{k}}\left(b_{k}\right)_{i}-1$, without loss of generality, let $s_{j}=m_{j}-1$.

By Eqs.(6) and (8), it gets

$$
\begin{aligned}
& \frac{\left(p^{\prime}-1\right) !\left(p-p^{\prime}\right) !}{p !} \\
= & \sum_{\substack{i \in B_{k} \\
t_{i} \wedge s_{k}, t-b_{k}=s-b_{k}}} \varphi_{i s_{i}}\left(N, m, \delta_{t}, \Gamma\right) \\
= & \sum_{i \in B_{k}^{\prime} \backslash j}\left(\varphi_{i m_{i}}\left(N, m, \delta_{t=s}, \Gamma\right)+\varphi_{i m_{i}}\left(N, m, \delta_{t=s \vee m_{j} e^{j}}, \Gamma\right)\right) \\
& +\varphi_{j\left(m_{j}-1\right)}\left(N, m, \delta_{t=s}, \Gamma\right) \\
= & \sum_{i \in B_{k}} \varphi_{i s_{i}}\left(N, m, \delta_{t=s}, \Gamma\right)+\sum_{i \in B_{k}^{\prime} \backslash j} \varphi_{i m_{i}}\left(N, m, \delta_{t=s \vee m_{j} e^{j}}, \Gamma\right) \\
= & \frac{\sum_{i \in B_{k}} h_{i s_{i}}^{B_{k}}\left(t \wedge b_{k}\right)}{h_{j\left(m_{j}-1\right)}^{B_{k}}\left(t \wedge b_{k}\right)} \varphi_{j\left(m_{j}-1\right)}\left(N, m_{i}, \delta_{t=s}, \Gamma\right) \\
& +\frac{\left(p^{\prime}-1\right) !\left(p-p^{\prime}\right) !}{p !} \sum_{i \in B_{k}^{\prime} \backslash j} h_{i m_{i}}^{B_{k}}\left(b_{k}\right) \\
& \frac{\frac{\left(p^{\prime}-1\right) !\left(p-p^{\prime}\right) !}{p !} \sum_{i \in B_{k}} h_{i s_{i}}^{B_{k}}\left(t \wedge b_{k}\right)}{\frac{\left(p^{\prime}-1\right) !\left(p-p^{\prime}\right) !}{p !} h_{j\left(m_{j}-1\right)}^{B_{k}}\left(t \wedge b_{k}\right)} \varphi_{j\left(m_{j}-1\right)}\left(N, m, \delta_{t=s}, \Gamma\right) \\
& \frac{\left(p^{\prime}-1\right) !\left(p-p^{\prime}\right) !}{p !} \sum_{i \in B_{k}^{\prime} \backslash j} h_{i m_{i}}^{B_{k}}\left(b_{k}\right) .
\end{aligned}
$$

Since

$$
\begin{aligned}
& \sum_{i \in \dot{B}_{k}} h_{i s_{i}}^{B_{k}}\left(t \wedge b_{k}\right)+\sum_{\substack{i \in \dot{B}_{k} s \wedge b_{k} \leq t \wedge b_{k}, s \wedge b_{k} \neq t \wedge b_{k} \\
t_{i}=s_{i}, t-b_{k}=s-b_{k}}} h_{i s_{i}}^{B_{k}}\left(t \wedge b_{k}\right) \\
& =\sum_{i \in \dot{B}_{k}} \sum_{\substack{s \wedge b_{k} \leq t \wedge b_{k}, t_{i}=s_{i}, t-b_{k}=s-b_{k}}} h_{i s_{i}}^{B_{k}}\left(t \wedge b_{k}\right) \\
& =1 \text {, }
\end{aligned}
$$

it has

$$
\varphi_{j s_{j}}\left(N, m, \delta_{t}, \Gamma\right)=\frac{\left(p^{\prime}-1\right) !\left(p-p^{\prime}\right) !}{p !} h_{j s_{j}}^{B_{k}}\left(t \wedge b_{k}\right) .
$$

From induction and $t_{i}=s_{i}$, it gets

$\varphi_{j t_{j}}\left(N, m, \delta_{t}, \Gamma\right)=\left\{\begin{array}{c}\frac{\left(p^{\prime}-1\right) !\left(p-p^{\prime}\right) !}{p !} h_{j t_{j}}^{B_{k}}\left(t \wedge b_{k}\right) \\ 0 \text { otherwise }\end{array} t_{j} \in M_{j}^{+}\right.$.

On the other hand, by Eq.(4) it has

$\psi_{j t_{j}}\left(N, m, \delta_{t}, \Gamma\right)=\left\{\begin{array}{c}\frac{\left(p^{\prime}-1\right) !\left(p-p^{\prime}\right) !}{p !} h_{j t_{j}}^{B_{k}}\left(t \wedge b_{k}\right) \\ 0 \quad \text { otherwise }\end{array} t_{j} \in M_{j}^{+}\right.$.

Namely, $\varphi$ and $\psi$ coincides on $\left(N, m, \delta_{T}, \Gamma\right)$. The proof is finished.

Remark 4.3. From Theorem 4.2, we know Eq.(4) is the unique coalitional value on $M C^{C N}$ that satisfies $\boldsymbol{A D D}, \boldsymbol{C}$, $\boldsymbol{C A}$ and $\boldsymbol{S Q}$, as well as the unique coalitional value on $M C^{C N}$ that satisfies $\boldsymbol{L}, \boldsymbol{C}, \boldsymbol{C A}$ and $\boldsymbol{S Q}$.

Young [25] proposed a characterization of the Shapley value by using strongly monotonicity. According to the literature [25], we propose strongly monotonicity on $M C^{C N}$ as follows:

strongly monotonicity (SMON). Let $v, w \in M C^{C N}$ and $i \in N$. If

$v\left(s \vee j e^{i}\right)-v\left(s \vee(j-1) e^{i}\right) \geq w\left(s \vee j e^{i}\right)-w\left(s \vee(j-1) e^{i}\right)$

for all $s \in F e(N, m, \Gamma)$ with $s_{i}=0$ and $j \in M_{i}^{+}$, then

$$
\varphi_{i j}(N, m, v, \Gamma) \geq \varphi_{i j}(N, m, w, \Gamma) .
$$

Similar to the property of symmetry given by Shapley [21] and the property of symmetric in the unions introduced by Owen [12], we introduce the veto property on $M C^{C N}$ as follows:

veto property $(\boldsymbol{V P})$. Let $v \in M C^{C N}$. For $B_{k} \in \Gamma$ and all $i_{1}, i_{2} \in T \cap B_{k}$, if $j_{1}=t_{i_{1}} \in M_{i_{1}}^{+}$and $j_{2}=t_{i_{2}} \in M_{i_{2}}^{+}$, then

$$
\begin{aligned}
& \sum_{\substack{x_{i_{2}}=j_{2}, t \wedge b_{k} \leq x \leq b_{k},}} h_{i_{2} j_{2}}^{B_{k}}(x) \varphi_{i_{1} j_{1}}(N, m, v, \Gamma) \\
= & \sum_{\substack{x_{i_{1}}=j_{1}, t \wedge b_{k} \leq x \leq b_{k},}} h_{i_{1} j_{1}}^{B_{k}}(x) \varphi_{i_{2} j_{2}}(N, m, v, \Gamma),
\end{aligned}
$$

where $t \in F e(N, m, \Gamma)$ is a veto coalition, and $T=\left\{i \in N \mid t_{i} \neq 0\right\}$. Here, $t \in F e(N, m, \Gamma)$ is said to be a veto coalition if it satisfies

$$
v(s)=\left\{\begin{array}{cc}
v(t) & s \in F e(N, m, \Gamma) \\
0 & \text { otherwise }
\end{array} .\right.
$$

Theorem 4.3. A coalitional value $\varphi$ satisfies $\boldsymbol{E F F}$, $S M O N, V P$ and $S \boldsymbol{Q}$ if, and only if, it is equal to Eq.(4), i.e., $\varphi_{i j}(N, m, v, \Gamma)=\psi_{i j}(N, m, v, \Gamma)$ for all $i \in N$ and all $j \in M_{i}^{+}$, where $v \in M C^{C N}$.

Proof. From Theorem 4.1 and Eq.(4), It is known that $\psi$ satisfies $E F F, S M O N$ and $S Q$.

In the following, we shall show Eq.(4) satisfies $\boldsymbol{V P}$. For any $t \in F e(N, m, \Gamma)$, without loss of generality, let 
$t=\vee_{l \in P_{t} \backslash k} b_{l} \vee s$ such that $s \leq b_{k}$, where $P_{t}=\left\{l \in P \mid t \wedge b_{l} \neq 0\right\}$. Then,

$$
\begin{aligned}
& \psi_{i_{1} j_{1}}(N, m, v, \Gamma) \\
= & \sum_{R \subseteq P \backslash k x \leq b_{k}, x_{i_{1}}=j_{1}} \frac{r !(p-r-1) !}{p !} h_{i_{1} j_{1}}^{B_{k}}(x) \\
& \times v\left((x \vee y)-v\left(x \vee y-e^{i_{1}}\right)\right) \\
= & \sum_{P_{t} \backslash k \subseteq R} \sum_{\substack{\leq \leq x \leq b_{k} \\
x_{i_{1}}=j_{1}}} \frac{r !(p-r-1) !}{p !} h_{i_{1} j_{1}}^{B_{k}}(x) v\left(s \vee\left(\vee_{l \in R \subseteq P \backslash k} b_{l}\right)\right) \\
= & \sum_{\substack{P_{t} \backslash k \subseteq R \\
\subseteq P \backslash k}} \frac{r !(p-r-1) !}{p !} \sum_{\substack{s \leq x \leq b_{k}, x_{i_{1}}=j_{1}}} h_{i_{1} j_{1}}^{B_{k}}(x) v\left(s \vee\left(\vee_{l \in R \subseteq P \backslash k} b_{l}\right)\right) \\
= & \frac{1}{p_{t}} \sum_{\substack{s \leq x \leq b_{k}, x_{i_{1}}=j_{1}}} h_{i_{1} j_{1}}^{B_{k}}(x) v(t) .
\end{aligned}
$$

where $y=\vee_{w \in R} b_{w}$, and $p_{t}$ is the cardinality of $P_{t}$.

For the same reason, it has

$$
\psi_{i_{2} j_{2}}(N, m, v, \Gamma)=\frac{1}{p_{t}} \sum_{s \leq x \leq b_{k}, x_{i_{2}}=j_{2}} h_{i_{2} j_{2}}^{B_{k}}(x) v(t) .
$$

Now one easily verifies that $\psi$ indeed satisfies the property of $\boldsymbol{V P}$.

We now prove the uniqueness part. Form $\boldsymbol{S M O N}$, it gets if $v\left(s \vee j e^{i}\right)-v\left(s \vee(j-1) e^{i}\right)=w\left(s \vee j e^{i}\right)-w\left(s \vee(j-1) e^{i}\right)$ for all $s \in F e(N, m, \Gamma)$, and all $i \in N$, where $s_{i}=0$ and $j \in M_{i}^{+}$, then

$$
\varphi_{i j}(N, m, v, \Gamma)=\varphi_{i j}(N, m, w, \Gamma) .
$$

Next consider the symmetric game $w$ on $(N, m, \Gamma)$ which is identically zero on $F e(N, m, \Gamma)$. Thus $w\left(s \vee j e^{i}\right)-w\left(s \vee(j-1) e^{i}\right)=0$ for all $s \in F e(N, m, \Gamma)$, all $i \in N$ and all $j \in M_{i}^{+}$, where $s_{i}=0$. Since, any $t \in F e(N, m, \Gamma)$ is a veto coalition, from $\boldsymbol{E F F}, \boldsymbol{V P}$ and $S \boldsymbol{Q}$, it has $\varphi_{i j}(N, m, w, \Gamma)=0$ for all $i \in N$ and all $j \in M_{i}^{+}$. From Eq.(9), it has $\varphi_{i j}(N, m, w, \Gamma)=0$ for all $i \in N$ and all $j \in M_{i}^{+}$. Namely, $\varphi$ satisfies $\boldsymbol{N P}$.

According to the proof of Lemma 3.1 in Derks and Peters [5], we know for all $v \in M C^{C N}, v$ can be expressed by

$$
v=\sum_{t \in F e(N, m, \Gamma)} \alpha_{t} u_{t}
$$

where $t$ is an analogue of unanimity game as given above. By Eq.(4), it gets

$\psi_{i j}\left(N, m, u_{s}, \Gamma\right)=\left\{\begin{array}{c}\frac{1}{p_{t}} \sum_{t \wedge b_{k} \leq x \leq b_{k},} h_{i j}^{B_{k}}(x) \quad j=t_{i} \in M_{i}^{+} \\ 0 \quad \text { otherwise }\end{array}\right.$

for any $B_{k} \in \Gamma$, where $p_{t}$ is the cardinality of $P_{t}=\{l \in$ $\left.P \mid t \wedge b_{l} \neq 0\right\}$.

Define the index $I$ of $v$ to be the minimum number of nonzero terms in some expression for $v$ of form (10). As in
Young [25], the theorem is proved by induction on $I$.

Case (i). If $I=0$, every $j \in M_{i}^{+}$is a "null level" for all $i \in N$, from above, it has

$$
\varphi_{i j}(N, m, w, \Gamma)=0 .
$$

Case (ii). If $I=1$, without loss of generality, let $v=\alpha_{t} u_{t}$ for some $t \in \mathrm{Fe}(N, m, \Gamma)$ where $\alpha_{t} \neq 0$. From above, it has $\varphi_{i j}(N, m, w, \Gamma)=0$ for all $i \in N$ and all $j \in M_{i}^{+}$with $j \neq t_{i}$. When $i \in T=\left\{i \in N \mid t_{i} \neq 0\right\}$, since $t$ is a veto coalition, from $\boldsymbol{E F F}, \boldsymbol{V P}$ and $\boldsymbol{S Q}$, it has

$$
\varphi_{i j}(N, m, v, \Gamma)=\frac{\alpha_{t}}{p_{t}} \sum_{\substack{t \wedge b_{k} \leq x \leq b_{k} \\ x_{i}=t_{i}=j}} h_{i j}^{B_{k}}(x),
$$

where $i \in B_{k} \in \Gamma$.

Therefore $\psi=\varphi$, whenever the index of $v$ is 0 or 1 .

Assume now that $\psi=\varphi$, whenever the index of $v$ is at most $I$, and let $v$ have index $I+1$ with expression

$$
v=\sum_{r=1}^{I+1} \alpha_{t_{r}} u_{t_{r}}
$$

where $\alpha_{t_{r}} \neq 0, t_{r} \in \mathrm{Fe}(N, m, \Gamma)$ and $p_{t_{r}}$ is the cardinality of $P_{t_{r}}=\left\{l \in P \mid t_{r} \wedge b_{l} \neq 0\right\}$ for all $r=1,2, \ldots, I+1$.

Let $t=\wedge_{r=1}^{I+1} t_{r}$, for any $i \in N \backslash T$ or any $i \in T$ with $\left(t_{r}\right)_{i}=$ $t_{i} \neq j \in M_{i}^{+}$for all $r=1,2, \ldots, I+1$.

Construct the game

$$
w=\sum_{r:\left(t_{r}\right)_{i} \neq 0} \alpha_{t_{r}} u_{t_{r}}
$$

where $T=\left\{i \in N \mid t_{i} \neq 0\right\}$.

The index of $w$ is at most $I$, since $v\left(s \vee j e^{i}\right)-v(s \vee(j-$ 1) $\left.e^{i}\right)=w\left(s \vee j e^{i}\right)-w\left(s \vee(j-1) e^{i}\right)$ for all $s \in F e(N, m, \Gamma)$, where $s_{i}=0$. By induction and $\mathbf{S M O N}$, it has

$$
\begin{aligned}
\varphi_{i j}(N, m, w, \Gamma) & =\varphi_{i j}(N, m, v, \Gamma) \\
& =\sum_{r:\left(t_{r}\right)_{i} \neq 0} \frac{\alpha_{t_{r}}}{p_{t_{r}}} \sum_{t_{r} \wedge b_{k} \leq x \leq b_{k}, x_{i}=\left(t_{r}\right)_{i}=j} h_{i j}^{B_{k}}(x) \\
& =\psi_{i j}(N, m, v, \Gamma) .
\end{aligned}
$$

For any $i \in T$ with $\left(t_{r}\right)_{i}=t_{i}=j \in M_{i}^{+}$and all $r=1,2, \ldots, I+1, t_{r}$ is a veto coalition for $\alpha_{t_{r}} u_{t_{r}}$, where $r=1,2, \ldots, I+1$.

From $\boldsymbol{E F F}, \boldsymbol{V P}$ and $S Q$, it gets

$$
\begin{aligned}
\varphi_{i j}(N, m, v, \Gamma) & =\sum_{r=1}^{I+1} \frac{\alpha_{t_{r}}}{p_{t_{r}}} \sum_{t_{r} \wedge b_{k} \leq x \leq b_{k}, x_{i}=\left(t_{r}\right)_{i}=j} h_{i j}^{B_{k}}(x) \\
& =\psi_{i j}(N, m, v, \Gamma) .
\end{aligned}
$$

Remark 4.4. As Young [25] pointed, strongly monotonicity can be replaced by independence condition, namely, a player's value depends only on the vector of his marginal contributions. Khmelnitskaya and Yanovskaya [18] proposed a characterization of the Owen coalition 
value by extending Owen's original axioms and replacing additivity with marginilist ( $\boldsymbol{M})$ (i.e. independence condition). From Theorem 4.3, we know Eq.(4) is the unique coalitional value on $M C^{C N}$ that satisfies $\boldsymbol{E F F}, \boldsymbol{M}$, $V P$ and $S Q$.

Property 4.1. Let $v \in M C^{C N}$. If $v(s)-v\left(s-(j-1) e^{i}\right)$ $=v\left(j e^{i}\right)$ for all $i \in N$ and all $s \in F e(N, m, \Gamma)$ with $j=s_{i} \in M_{i}^{+}$, then $\psi_{i j}(N, m, v, \Gamma)=v\left(j e^{i}\right)$.

Proof. By Eq.(4), it has

$$
\begin{aligned}
& \psi_{i j}(N, m, v, \Gamma) \\
= & \sum_{R \subseteq P \backslash k s \leq b_{k}, s_{i}=j} \frac{r !(p-r-1) !}{p !} h_{i j}^{B_{k}}(s) \\
& \times\left(v(s \vee t)-v\left(s \vee t-e^{i}\right)\right) \\
= & \sum_{R \subseteq P \backslash k s \leq b_{k}, s_{i}=j} \frac{r !(p-r-1) !}{p !} h_{i j}^{B_{k}}(s) v\left(j e^{j}\right) \\
= & \sum_{R \subseteq P \backslash k} \frac{r !(p-r-1) !}{p !} \sum_{s \leq b_{k}, s_{i}=j} h_{i j}^{B_{k}}(s) v\left(j e^{j}\right) \\
= & \sum_{R \subseteq P \backslash k} \frac{r !(p-r-1) !}{p !} v\left(j e^{j}\right) \\
= & v\left(j e^{j}\right) .
\end{aligned}
$$

Player $i \in N$ is called a dummy player, if for all $j \in M_{i}^{+}$and all $s \in F e(N, m, \Gamma)$ with $s_{i}=0$, it has $v\left(s \vee j e^{i}\right)-v\left(s-\left(j^{1}\right) e^{i}\right)=v\left(j e^{i}\right)$. If $i \in N$ is a dummy player, then $\psi_{i j}(N, m, v, \Gamma)=v\left(j e^{i}\right)$ for all $j \in M_{i}^{+}$.

Property 4.2. Let $v \in M C^{C N}$ and any $s \in F e(N, m, \Gamma)$ with $s_{i}=j \in M_{i}^{+}$. If $v(s)-v\left(s-e^{i}\right)=v\left(j e^{i}\right)-$ $v\left((j-1) e^{i}\right)$, then $\psi_{i j}(N, m, v)=v\left(j e^{i}\right)-v\left((j-1) e^{i}\right)$.

Proof. The proof of Property 4.2 is similar to that of Property 4.1.

From Property 4.2, we know if $v(s)-v\left(s-e^{i}\right)$ $=v\left(j e^{i}\right)-v\left((j-1) e^{i}\right)$ holds for all $s \in F e(N, m, \Gamma)$ and all $j \in M_{i}^{+}$with $s_{i}=j$, then $\sum_{j=1}^{m_{i}} \psi_{i j}(N, m, v, \Gamma)=v\left(m_{i} e^{i}\right)$.

\section{The relationship between the given coalitional value and the core}

Definition 5.1. The multichoice game $(N, m, v)$ is called convex if it satisfies

$$
v(s \vee t)+v(s \wedge t) \geq v(s)+v(t)
$$

for all $s, t \in M$.

When we restrict the multichoice game $(N, m, v)$ in the setting of traditional games, Definition 5.1 reduces to be the concept of convexity for traditional case. Similar to the definition of the core of games with a coalition structure, we define the core of multichoice games with a coalition structure as follows:

Definition 5.2. The core $C(N, m, v, \Gamma)$ of $v \in M C^{C N}$ is denoted by

$$
\begin{gathered}
C(N, m, v, \Gamma)=\left\{x \mid \sum_{i \in S j=1} \sum_{i}^{s_{i}} x_{i j} \geq v(s), \forall s \in F e(N, m, \Gamma),\right. \\
\left.\sum_{i \in N} \sum_{j=1}^{m_{i}} x_{i j}=v(m)\right\},
\end{gathered}
$$

where $S=\left\{i \mid s_{i} \neq 0, i \in N\right\}$.

Theorem 5.1. Let $v \in M C^{C N}$ be convex, then $C(N, m, v, \Gamma) \neq \emptyset$.

Proof. Let $\Gamma=\left\{B_{1}, B_{2}, \ldots, B_{h}\right\}$, we rearrange the order of the players in $(N, m, \Gamma)$, and get $\pi \Gamma=\left\{\pi B_{1}, \pi B_{2}, \ldots\right.$, $\left.\pi B_{h}\right\}$ such that $\pi B_{1}=\left\{i_{1}, i_{2}, \ldots, i_{d_{1}}\right\}, \pi B_{2}=\left\{i_{d_{1}+1}\right.$, $\left.i_{d_{1}+2}, \ldots, i_{d_{1}+d_{2}}\right\}, \ldots, \pi B_{m}=\left\{i_{\sum_{l=1}^{h-1} d_{l}+1}, i_{\sum_{l=1}^{h-1} d_{l}+2}, \ldots, i_{n}\right\}$, where $d_{i}$ denotes the cardinality of $B_{i}$ for all $i \in\{1,2, \ldots, h\}$. Let

$$
\begin{aligned}
& x_{i_{1} 1}=v\left(e^{i_{1}}\right), \ldots, x_{i_{1} m_{i_{1}}}=v\left(m_{i_{1}} e^{i_{1}}\right)-v\left(\left(m_{i_{1}}-1\right) e^{i_{1}}\right), \ldots, \\
& x_{i_{d_{1}} 1}=v\left(\vee_{l=i_{1}}^{d_{1}-1} m_{l} e^{l} \vee e^{d_{1}}\right)-v\left(\bigvee_{l=i_{1}}^{d_{1}-1} m_{l} e^{l}\right), \ldots, \\
& x_{i_{d_{1}} m_{d_{1}}}=v\left(\vee_{l=i_{1}}^{d_{1}} m_{l} e^{l}\right)-v\left(\vee_{l=i_{1}}^{d_{1}-1} m_{l} e^{l} \vee\left(m_{d_{1}}-1\right) e^{d_{1}}\right), \ldots, \\
& x_{i_{d_{1}}+1}=v\left(\vee_{l=i_{1}}^{d_{1}} m_{l} e^{l} \vee e^{d_{1}+1}\right)-v\left(\bigvee_{l=i_{1}}^{d_{1}} m_{l} e^{l}\right), \ldots, \\
& x_{i_{n} 1}=v\left(m-\left(m_{i_{n}}-1\right) e^{i_{n}}\right)-v\left(m-m_{i_{n}} e^{i_{n}}\right), \ldots, \\
& x_{i_{n} m_{i_{n}}}=v(m)-v\left(m-e^{n}\right) .
\end{aligned}
$$

It is obvious that $\sum_{i \in N} \sum_{j=1}^{m_{i}} x_{i j}=v(m)$ holds.

For any $s \in \mathrm{Fe}(N, m, \Gamma)$, without loss of generality, let $s=$ $\vee_{l \in R \subseteq P \backslash k} b_{l} \vee t$ with $t \leq b_{k}$. If $t \leq b_{k}$ and $t \neq b_{k}$, then it has

$$
m-t=\vee_{l \in P \backslash(R \cup k)} b_{l} \vee b_{k}-t \wedge b_{k} .
$$

Let $\left\{j_{1}, j_{2}, \ldots, j_{d}\right\}=B_{k}$ such that $j_{1} \leq j_{2} \leq \ldots \leq j_{d}$. From $t \leq b_{k}$ and $t \neq b_{k}$, without loss of generality, let $\left\{j_{t}, j_{t+1}, \ldots, j_{t+a}\right\} \subseteq B_{k}$, where $t_{j_{c}} \neq m_{j_{c}}$ for all $c \in\{t, t+1, \ldots, t+a\}$. According to above, we rearrange the order of the players in $(N, m, \Gamma)$ such that $s=\vee_{l \in\{1,2, \ldots k-1\} \subseteq P \backslash k} \pi b_{l} \quad \vee \quad t, \quad$ where $\pi b_{l}=\left\{m_{\sum_{\Sigma_{x=1}^{l}}^{l} d_{x-1}+1}, m_{\Sigma_{\Sigma_{x=1}^{l}}^{l} d_{x-1}+2}, \ldots m_{\Sigma_{\Sigma_{x=1}^{l+1} d_{x-1}}}\right\}$ for all $l \in\{1,2, \ldots, k-1\}$. Let

$$
r=\vee_{l \in\{1,2, \ldots k-1\} \subseteq P \backslash k} \pi b_{l} \bigvee_{a=1}^{t-1} m_{j_{a}} e^{j_{a}} \vee\left(s_{j_{t}}+1\right) e^{j_{t}},
$$

then it gets

$$
r \vee s=s \vee\left(s_{j_{t}}+1\right) e^{j_{t}}, \quad r \wedge s=r-e^{j_{t}} .
$$

From the convexity of $v$, it gets

$$
v\left(s \vee\left(s_{j_{t}}+1\right) e^{j_{t}}\right)+v\left(r-e^{j_{t}}\right) \geq v(s)+v(r)
$$

and

$$
\begin{aligned}
x_{j_{t}\left(s_{j_{t}}+1\right)} & =v(r)-v\left(r-e^{j_{t}}\right) \\
& \leq v\left(s \vee\left(s_{j_{t}}+1\right) e^{j_{t}}\right)-v(s) .
\end{aligned}
$$


Namely,

$$
\begin{aligned}
& \left(\sum_{i \in S j=1} \sum_{i}^{s_{i}} x_{i j}+x_{j_{t}\left(s_{j_{t}}+1\right)}\right)-\sum_{i \in S} \sum_{j=1}^{s_{i}} x_{i j} \\
& \leq v\left(s \vee\left(s_{j_{t}}+1\right) e^{j_{t}}\right)-v(s),
\end{aligned}
$$

where $S=\left\{k \mid s_{k} \neq 0, k \in N\right\}$. Thus,

$$
\begin{aligned}
& \left(\sum_{i \in S} \sum_{j=1}^{s_{i}} x_{i j}+x_{j_{t}\left(s_{j_{t}}+1\right)}\right)-v\left(s \vee\left(s_{j_{t}}+1\right) e^{j_{t}}\right) \\
& \leq \sum_{i \in S} \sum_{j=1}^{s_{i}} x_{i j}-v(s) .
\end{aligned}
$$

Repeat the above process $\sum_{i \in N} m_{i}-\sum_{i \in N} s_{i}$ times, it has

$$
\sum_{i \in S} \sum_{j=1}^{s_{i}} x_{i j}-v(s) \geq \sum_{i \in N} \sum_{j=1}^{m_{i}} x_{i j}-v(m)=0 .
$$

From Definition 5.1, it shows $x \in C(N, m, v, \Gamma)$.

If $t=b_{k}$, then let $r=\vee_{l \in\{1,2, \ldots k-1, k\} \subseteq P} \pi b_{l} \vee e^{j}$, where $j \in B_{k+1}$ and $k+1 \in P$. Repeat the above process, one can also get $x \in C(N, m, v, \Gamma)$.

Theorem 5.2. Let $v \in M C^{C N}$ be convex, then $\psi(N, m, v, \Gamma) \in C(N, m, v, \Gamma)$.

Proof. From Eq.(4) and Theorem 5.1, we know the function $\psi(N, m, v, \Gamma)$ is a convex combination of $h \sum_{k \in P}\left(\sum_{i \in B_{k}} m_{i}\right) ! / \prod_{i \in B_{k}} m_{i}$ ! elements in $C(N, m, v, \Gamma)$. Thus, $\psi(N, m, v, \Gamma) \in C(N, m, v, \Gamma)$ for $C(N, m, v, \Gamma)$ being a convex set. $\square$

Remark 5.1. The relationship between the given coalitional value and the core coincides with that of the Shapley value and the core of traditional case. The proof of Theorem 5.1 is an extension of the classical case [26]. Example 5.1. Let $N=\{1,2,3,4\}, m=(2,3,2,1)$ and $\Gamma=\left\{B_{1}, B_{2}\right\}$, where $B_{1}=\{1,2\}$ and $B_{2}=\{3,4\}$. The coalition values for $v \in M C^{C N}$ are given by Table 1 .

Table 1: Values for coalitions

\begin{tabular}{cccccc}
\hline$S_{0}$ & $v_{0}\left(S_{0}\right)$ & $S_{0}$ & $v_{0}\left(S_{0}\right)$ & $S_{0}$ & $v_{0}\left(S_{0}\right)$ \\
\hline$(1,0,0,0)$ & 1 & $(2,1,0,0)$ & 8 & $(2,3,1,0)$ & 18 \\
$(0,1,0,0)$ & 1 & $(2,1,0,0)$ & 8 & $(2,3,2,0)$ & 20 \\
$(0,0,1,0)$ & 1 & $(2,2,0,0)$ & 10 & $(1,1,2,1)$ & 15 \\
$(0,0,0,1)$ & 1 & $(1,3,0,0)$ & 10 & $(1,2,2,1)$ & 20 \\
$(2,0,0,0)$ & 2 & $(2,3,0,0)$ & 15 & $(1,3,2,1)$ & 25 \\
$(0,2,0,0)$ & 2 & $(1,0,2,1)$ & 10 & $(2,1,2,1)$ & 20 \\
$(0,0,2,0)$ & 2 & $(2,0,2,1)$ & 12 & $(2,3,1,1)$ & 20 \\
$(0,3,0,0)$ & 3 & $(0,1,2,1)$ & 12 & $(2,2,2,1)$ & 30 \\
$(1,1,0,0)$ & 5 & $(0,2,2,1)$ & 15 & $(2,3,2,1)$ & 40 \\
$(0,0,1,1)$ & 5 & $(0,3,2,1)$ & 18 & & \\
$(1,2,0,0)$ & 8 & $(2,3,0,1)$ & 15 & & \\
\hline
\end{tabular}

By Eq.(4), it has

$$
\psi_{11}(N, m, v, \Gamma)=3.45,
$$

$$
\begin{aligned}
& \psi_{12}(N, m, v, \Gamma)=6.75, \\
& \psi_{21}(N, m, v, \Gamma)=3.55, \\
& \psi_{22}(N, m, v, \Gamma)=4, \\
& \psi_{23}(N, m, v, \Gamma)=5.75, \\
& \psi_{31}(N, m, v, \Gamma)=2.83, \\
& \psi_{32}(N, m, v, \Gamma)=8.17, \\
& \psi_{41}(N, m, v, \Gamma)=5.5
\end{aligned}
$$

and

$$
\sum_{i=1}^{4} \sum_{j=1}^{m_{i}} \psi_{i j}(N, m, v, \Gamma)=40=v(m) .
$$

From Definition 5.1, we know the game $v \in M C^{C N}$, given in example 5.1, is convex. Thus, $\psi(N, m, v, \Gamma)$ is an element in the core.

\section{Conclusion}

We have introduced and researched a coalitional value for multichoice games with a coalition structure, which can be regarded as an extension of the Owen value [12] and the Shapley value [2]. Furthermore, we discuss three characterizations of the given coalitional value, which will help us better understand it. Like other solutions, we can have other axiomatic systems to define the given coalitional value. The introduced model for multichoice games is different to any existing one. Because of the advantages of the value proposed by van den Nouweland et al. [2], the given coalitional value considers the player participation levels, and endows the different weights with respect to the different participation levels.

However, we mainly study one coalitional value for multichoice games with a coalition strucutre, and it will be interesting to discuss other payoff indices. Furthermore, we shall study the payoff indices of multichoice games under precedence constraints by using graph theory $[27,28,29,30,31,32]$.

\section{Acknowledgement}

The authors are grateful to two anonymous referees for a careful checking of the details and for helpful comments that improved this paper.

\section{References}

[1] C. Hsiao and T. Raghavan, Shapley value for multi-choice cooperative games (I), Games Econ. Behav., 5, 240-256 (1993).

[2] A. van den Nouweland, S. Tijs, J. Potters and J. Zarzuelo, Cores and related solution concepts for multi-choice games, ZOR-Math. Meth. Oper. Res., 41, 289-311 (1995). 
[3] E. Calvo and J. C. Santos, The multichoice value, Working Paper, Department of Applied Economics, University of Pais Vasco, Spain, (1997).

[4] F. Klijn, M. Slikker and J. Zarzuelo, Characterizations of a multi-choice value, Int. J. Game Theory, 28, 521-532 (1999).

[5] J. Derks and H. Peters, A Shapley value for games with restricted coalitions, Int. J. Game Theory, 21, 351-360 (1993).

[6] E. Calvo and J. C. Santos, A value for multichoice games, Math. Soc. Sci., 40, 341-354 (2000).

[7] H. Peters and H. Zank, The egalitarian solution for multichoice games, Ann. Oper. Res., 137, 399-409 (2005).

[8] B. Dutta and D. Ray, A concept of egalitarianism under participation constraints, Econometrica, 57, 615-635 (1989).

[9] Y. A. Hwang and Y. H. Liao, Potential approach and characterizations of a Shapley value in multi-choice games, Math. Soc. Sci., 56, 321-335 (2008).

[10] Y. A. Hwang and Y. H. Liao, Equivalence theorem, consistency and axiomatizations of a multi-choice value, J. Global. Optim., 45, 597-613 (2009).

[11] R. J. Aumann and J. H. Drze, Cooperative games with a coalition structure, Int. J. Game Theory, 3, 217-237 (1974).

[12] G. Owen, Values of games with a priori unions. In: Henn, R., Moeschlin, O. (Eds.), Lecture Notes in Economics and Mathematical Systems. Essays in Honor of Oskar Morgenstern, Nueva York: Springer Verlag, 76-88 (1977).

[13] M. J. Albizuri, Axiomatizations of the Owen value without efficiency, Math. Soc. Sci., 55, 78-89 (2008).

[14] J. M. Alonso-Meijide and M. G. Fiestras-Janeiro, Modification of the Banzhaf value for games with a coalition structure, Ann. Oper. Res., 109, 213-227 (2002).

[15] J. M. Alonso-Meijide, F. Carreras, M. G. Fiestras-Janeiro and G. Owen, A comparative axiomatic characterization of the Banzhaf-Owen coalitional value, Decis. Support Syst., 43, 701-712 (2007).

[16] S. Hart and M. Kurz, Endogenous formation of coalitions, Econometrica, 51, 1047-1064 (1983).

[17] G. Hamiache, A new axiomatization of the Owen value for games with a coalition structure, Math. Soc. Sci., 37, 281-305 (1999).

[18] A. B. Khmelnitskaya and E. B. Yanovskaya, Owen coalitional value without additivity axiom. Math. Math. Oper. Res., 66, 255-261 (2007).

[19] G. Owen, Characterization of the Banzhaf-Coleman index, SIA M. J. Appl. Math., 35, 315-327 (1978).

[20] M. J. Albizuri, The multichoice coalition value, Ann. Oper. Res., 172, 363-374 (2009).

[21] L. S. Shapley, A value for n-person games, Ann. Math. Stud., 28, 307-318 (1953).

[22] M. A. Jones and J. M. Wilson, Two-step coalition values for multichoice games, Math. Math. Oper. Res., 77, 65-991 (2013).

[23] U. Faigle and W. Kern, The Shapley value for cooperative games under precedence constraints, Int. J. Game Theory, 21, 249-266 (1992).

[24] J. M. Bilbao, Axioms for the Shapley value on convex geometries, Eur. J. Oper. Res., 110, 368-376 (1998).

[25] H. Young, Monotonic solutions of cooperative games, Int. J. Game Theory, 14, 65-72 (1985).

[26] L. S. Shapley, Cores of convex games, Int. J. Game Theory, 1, 11-26 (1971).
[27] I. Saleem, H. Nawaz, I. Ahmed, S. M. Abbas, Analytical Evaluation of Tri-band Printed Antenna, Information Sciences Letters, 1, 85-89 (2012).

[28] C. Y. Zhou, H. J. Chen and J. H. Tao, A domain ontologydriven semantic graph auto extraction system, Appl. Math. Inf. Sci., 5, 9-16 (2011).

[29] B.W. Shivaraj, H. N. Narasimha Murthy, M, Krishna, S. C. Sharma, Investigation of Influence of Spin Coating Parameters on the Morphology of $\mathrm{ZnO}$ Thin Films by Taguchi Method, International Journal of Thin Films Science and Technology, 2, 143-154 (2013).

[30] B. Zhang and Z. Juan, Modeling User Eequilibrium and the Day-to-day Traffic Evolution based on Cumulative Prospect Theory, Information Science Letters, 2, 9-12 (2013).

[31] M. Iwundu and P. Chigbu, A HILL-CLIMBING COMBINATORIAL ALGORITHM FOR CONSTRUCTING N-POINT D-OPTIMAL EXACT DESIGNS, Journal of Statistics Applications \& Probability, 1, 133-146 (2012).

[32] N. Kanwal and A. Khan, Efficiency Analysis in Outsourced Database using One Time Signature Scheme, Information Science Letters, 2, 1-8 (2013).

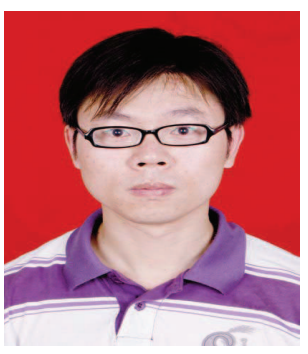

$\begin{array}{lll} & \text { Fanyong Meng is } \\ \text { a lecturer in School of }\end{array}$ Management at Qingdao Technological University, Qiangdao, China. He received the $\mathrm{PhD}$ degree in School of Management and Economics at Beijing Institute of Technology, Beijing, China. His research interests are in the areas of Optimization theory and methods, game theory and decision making. He has published over 40 research articles in reputed international journals of mathematical and information sciences. He is referee and editor of information journals.

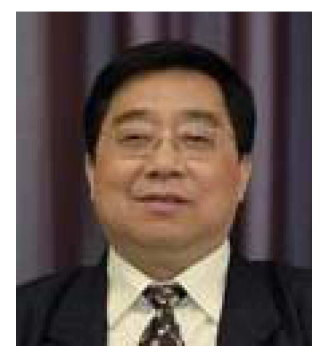

Qiang Zhang is
a professor in School of Management and Economics at Beijing Institute of Technology, Beijing, China. $\mathrm{He}$ received the $\mathrm{PhD}$ degree in School of Traffic and Transportation at Southwest Jiao Tong University, Chengdu, China. At present, he has contributed over 200 journal articles to reputed international journals of applied mathematics and Information sciences. He is an associate editor of Journal of Intelligent and Fuzzy Systems. His current research interests include management decisions in quantitative theory and method, the modern logistics and supply chain management, uncertain system theory and application. 